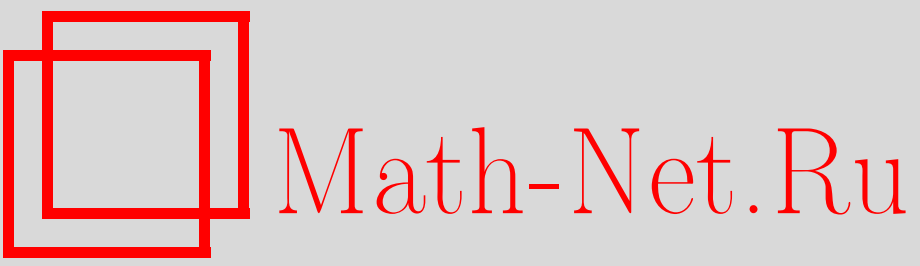

Р. Ф. Эфендиев, Спектральный анализ одного класса несамосопряженных дифференциальных операторных пучков с обобщенной функцией, ТMФ, 2005, том 145, номер 1, 102-107

DOI: https://doi.org/10.4213/tmf1883

Использование Общероссийского математического портала Math-Net.Ru подразумевает, что вы прочитали и согласны с пользовательским соглашением

http://www. mathnet.ru/rus/agreement

Параметры загрузки:

IP: 3.85 .7 .115

26 апреля 2023 г., 18:32:55 


\section{СПЕКТРАЛЬНЫЙ АНАЛИЗ ОДНОГО КЛАССА НЕСАМОСОПРЯЖКЕННЫХ ДИФФЕРЕНЦИАЛЬНЫХ ОПЕРАТОРНЫХ ПУЧКОВ С ОБОБЩЕННОЙ ФУНКЦИЕЙ}

Исследуется спектр, а также решается обратная задача для пучка несамосопряженных дифференциальных операторов второго порядка с обобщенной функцией в пространстве $L_{2}(-\infty,+\infty)$.

Ключевые слова: уравнение Шредингера, дельта-функция Дирака, решение Йоста, спектральные особенности, обратная задача.

\section{1. ВВЕДЕНИЕ}

Распространение волн в одномерной неконсервативной среде в частотной области описывается уравнением Шредингера

$$
y_{+}^{\prime \prime}(x, \lambda)+\lambda^{2} y_{+}(x, \lambda)=[i \lambda p(x)+q(x)] y_{+}(x, \lambda), \quad x \in \mathbb{R},
$$

где $\mathbb{R}$ - действительная ось, $\lambda$ - волновое число (известное как импульс), $\lambda^{2}$ - энергия, $p(x)$ - объединенный эффект поглошения и генерации энергии, $q(x)$ описывает восстановление плотности силы. В нестационарном случае оно соответствует волновому уравнению

$$
\frac{\partial^{2} u}{\partial x^{2}}-\frac{\partial^{2} u}{\partial t^{2}}-p(x) \frac{\partial u}{\partial t}=q(x) u, \quad t, x \in \mathbb{R},
$$

где волновая скорость равна 1 . Когда $p(x) \leqslant 0$, имеет место чистое поглощение; однако мы не будем фиксировать знак $p(x)$. Следовательно, важную роль играет и уравнение

$$
y_{-}^{\prime \prime}(x, \lambda)+\lambda^{2} y_{-}(x, \lambda)=[-i \lambda p(x)+q(x)] y_{-}(x, \lambda), \quad x \in \mathbb{R},
$$

где знак $p(x)$ уже изменен.

${ }^{*}$ Институт прикладной математики, Бакинский государственный университет, Баку, Азербайджан. E-mail: rakibaz@yahoo.com 
Приступим к исследованию спектра, а также к решению обратной задачи для пучка $L$ несамосопряженных дифференциальных операторов, порожденных формальным дифференциальным выражением

$$
l\left(\frac{d}{d x}, \lambda\right) \equiv-\frac{d^{2}}{d x^{2}}+2 \lambda p(x)+q(x)+\beta \delta(x)-\lambda^{2}
$$

с обобшенной функцией в пространстве $L_{2}(-\infty, \infty)$. Здесь $\delta(x)$ - дельта-функция Дирака, $\beta<0$ - вешественное число, $\lambda$ - комплексное число, а коэффициенты $p(x), q(x)$ имеют вид

$$
\begin{array}{ll}
p(x)=\sum_{n=1}^{\infty} p_{n} e^{i n x}, \quad \sum_{n=1}^{\infty} n\left|p_{n}\right|<\infty, \\
q(x)=\sum_{n=1}^{\infty} q_{n} e^{i n x}, \quad \sum_{n=1}^{\infty}\left|q_{n}\right|<\infty .
\end{array}
$$

В связи с важными приложениями в квантовой механике представляет интерес исследование спектральных характеристик операторного пучка $L$, а в случае, когда хотя бы одна из сталкиваюшихся частищ - фермион, становится актуальным и решение обратной задачи при наличии центрального и спин-орбитального потенциалов [1], [2]. Как правило, рассматриваемая задача связана с разрывными свойствами физических характеристик среды.

Отметим, что при $p(x)=0$ и выполнении условия

$$
\int_{-\infty}^{+\infty}\left(1+x^{2}\right) q(x) d x<\infty
$$

где $q(x)$ - скалярная вещественнозначная неотрицательная функция на $(-\infty,+\infty)$, спектральные характеристики данной задачи изучены в работе [3].

Обратная задача в случае $p(x)=0$ и при выполнении условия

$$
\int_{-\infty}^{+\infty}(1+|x|)|q(x)| d x<\infty
$$

где $q(x)$ - вешественнозначная исчезающая при $x<0$ функция, исследована в работе [4].

Случай $\beta=0$ рассмотрен в работе [5].

В разделе 2 в предположении, что потенциалы имеют вид (2), строятся соответствующие решения Йоста, вводится понятие обобшенного нормировочного числа и исследуется спектр операторного пучка $L$. В разделе 3 решается обратная задача восстановления потенциалов (2) по нормировочным числам. 


\section{2. ПОСТРОЕНИЕ РЕЗОЛЬВЕНТЫ И ИССЛЕДОВАНИЕ СПЕКТРА ОПЕРАТОРНОГО ПУЧКА $L$}

Решением уравнения $L(y)=0$ назовем решение системы

$$
\begin{gathered}
-y^{\prime \prime}(x)+2 \lambda p(x) y(x)+q(x) y(x)=\lambda^{2} y(x), \\
y(+0)=y(-0)=y(0), \\
y^{\prime}(+0)-y^{\prime}(-0)=-\beta y(0) .
\end{gathered}
$$

Известно [5], что для уравнения (3) справедлива

ТЕОрема 1. Пусть $p(x)$ и $q(x)$ имеют вид (2). Тогда уравнение (3) имеет спеииальные решения вида

$$
f_{ \pm}(x, \lambda)=e^{ \pm i \lambda x}\left(1+\sum_{n=1}^{\infty} \nu_{n}^{ \pm} e^{i n x}+\sum_{n=1}^{\infty} \sum_{\alpha=n}^{\infty} \frac{\nu_{n \alpha}^{ \pm}}{n \pm 2 \lambda} e^{i \alpha x}\right)
$$

где числа $\nu_{n}^{ \pm}$и $\nu_{n \alpha}^{ \pm}$определяются из следующих рекуррентньх соотношений:

$$
\begin{array}{r}
\alpha^{2} \nu_{\alpha}^{ \pm}+\alpha \sum_{n=1}^{\alpha} \nu_{n \alpha}^{ \pm}+\sum_{s=1}^{\alpha-1}\left(q_{\alpha-s} \nu_{s}^{ \pm}-p_{\alpha-s} \sum_{n=1}^{s} \nu_{n s}^{ \pm}\right)+q_{\alpha}=0 \\
\alpha(\alpha-n) \nu_{n \alpha}^{ \pm}+\sum_{s=n}^{\alpha-1}\left(q_{\alpha-s} \mp n p_{\alpha-s}\right) \nu_{n s}^{ \pm}=0 \\
\alpha \nu_{\alpha}^{ \pm} \pm \sum_{s=1}^{\alpha-1} \nu_{s}^{ \pm} p_{\alpha-s} \pm p_{\alpha}=0
\end{array}
$$

причем ряд (5) допускает дважды почленное дифферениирование.

Функции $f_{+}(x, \lambda)$ и $f_{-}(x, \lambda)$ линейно независимы, и их вронскиан $W\left[f_{+}(x, \lambda), f_{-}(x, \lambda)\right]=2 i \lambda$. Это вытекает из того факта, что $W$ не зависит от $x$, а функции $f_{ \pm}(x, \lambda)$ допускают голоморфные по $x$ продолжения в нижнюю и верхнюю полуплоскости, соответственно, и

$$
\lim _{\operatorname{Im} x \rightarrow \infty} f_{ \pm}^{(\nu)}(x, \lambda) e^{\mp i \lambda x}=( \pm i \lambda)^{\nu}, \quad \nu=\overline{0,1}
$$

Положим

$$
f_{n}^{ \pm}(x)=\lim _{\lambda \rightarrow \mp n / 2}(n \pm 2 \lambda) f_{ \pm}(x, \lambda)=\sum_{\alpha=n}^{\infty} V_{n \alpha}^{ \pm} e^{i \alpha x} e^{-i(n / 2) x} .
$$

Из соотношения (6б) следует, что если $V_{n n}^{+}=0$, то $V_{n \alpha}^{+}=0$ при всех $\alpha>n$ и $f_{n}^{+}(x) \equiv 0$ (аналогично для $\left.f_{n}^{-}(x)\right)$. Следовательно, точки $( \pm n / 2)$ не являются особыми точками для $f_{ \pm}(x, \lambda)$, соответственно. Тогда

$$
W\left[f_{n}^{ \pm}(x), f_{\mp}\left(x, \mp \frac{n}{2}\right)\right]=0
$$


и, следовательно, функции $f_{\mp}(x, \mp n / 2), f_{n}^{ \pm}(x)$, которые являются решениями уравнения $L(y)=0$ при $\lambda=\mp n / 2$, линейно зависимы. Отсюда

$$
f_{n}^{ \pm}(x)=S_{n}^{ \pm} f_{\mp}\left(x, \mp \frac{n}{2}\right) .
$$

Сравнение аналитических выражений этих функций показывает, что

$$
S_{n}^{ \pm}=V_{n n}^{ \pm}
$$

Разделим $\lambda$-плоскость на сектора $S_{\nu}=\{\nu \pi<\arg \lambda<(\nu+1) \pi\}, \quad \nu=0,1$, и переобозначим $f_{ \pm}(x, \lambda)$ таким образом, чтобы выполнялись условия $f_{+}(x, \lambda) \in L_{2}(0,+\infty)$, $f_{-}(x, \lambda) \in L_{2}(-\infty, 0)$. Отметим, что любое решение уравнения $(3) y(x, \lambda)$ представляется линейной комбинацией функций $f_{ \pm}(x, \lambda)$, и его можно записать в виде

$$
y(x, \lambda)= \begin{cases}c_{0}(x) f_{+}(x, \lambda)+c_{1}(x) f_{-}(x, \lambda), & x \in(-\infty, 0), \\ c_{2}(x) f_{+}(x, \lambda)+c_{3}(x) f_{-}(x, \lambda), & x \in(0,+\infty),\end{cases}
$$

где $c_{j}(x), j=\overline{0,3}$, такие, что для $y(x, \lambda)$ выполняются условия (4).

Построим резольвенту операторного пучка $L$ при $\lambda \in S_{\nu}$. Для этого решается задача

$$
\begin{gathered}
-y^{\prime \prime}(x)+2 \lambda p(x) y(x)+q(x) y(x)=\lambda^{2} y(x)+f(x), \quad x \neq 0, \\
y(+0)=y(-0)=y(0), \\
y^{\prime}(+0)-y^{\prime}(-0)=-\beta y(0)
\end{gathered}
$$

в $L_{2}(-\infty,+\infty)$. Здесь $f(x)-$ произвольная функция, принадлежащая $L_{2}(-\infty,+\infty)$. Для нахождения функций $c_{j}(x), j=\overline{0,1}$, составим систему уравнений

$$
\begin{aligned}
& c_{0}^{\prime}(x) f_{+}(x, \lambda)+c_{1}^{\prime}(x) f_{-}(x, \lambda)=0, \\
& c_{0}^{\prime}(x) f_{+}^{\prime}(x, \lambda)+c_{1}^{\prime}(x) f_{-}^{\prime}(x, \lambda)=f(x),
\end{aligned}
$$

из которой имеем

$$
\begin{aligned}
& c_{0}(x)=-\frac{1}{2 i \lambda} \int_{-\infty}^{x} f_{-}(t, \lambda) f(t) d t+c_{0}, \\
& c_{1}(x)=-\frac{1}{2 i \lambda} \int_{x}^{0} f_{+}(t, \lambda) f(t) d t+c_{1},
\end{aligned}
$$

где $x \in(-\infty, 0), c_{j}, j=\overline{0,1},-$ произвольные числа. Проведя аналогичные вычисления для функций $c_{j}(x), j=\overline{2,3}$, получим, что решение уравнения (8) при $\lambda \in S_{\nu}$ имеет вид

$$
y(x, \lambda)=-\frac{1}{2 i \lambda}\left\{\begin{array}{c}
-\int_{-\infty}^{x} f_{+}(x, \lambda) f_{-}(t, \lambda) f(t) d t-\int_{x}^{0} f_{-}(x, \lambda) f_{+}(t, \lambda) f(t) d t+ \\
+c_{0} f_{+}(x, \lambda)+c_{1} f_{-}(x, \lambda), \quad x \in(-\infty, 0), \\
-\int_{0}^{x} f_{+}(x, \lambda) f_{-}(t, \lambda) f(t) d t-\int_{x}^{\infty} f_{-}(x, \lambda) f_{+}(t, \lambda) f(t) d t+ \\
+c_{2} f_{+}(x, \lambda)+c_{3} f_{-}(x, \lambda), \quad x \in(0,+\infty) .
\end{array}\right.
$$


В силу условия $y(\cdot, \lambda) \in L_{2}(-\infty,+\infty), f_{+}(x, \lambda) \in L_{2}(0, \infty), \quad f_{-}(x, \lambda) \in L_{2}(-\infty, 0)$ определяем, что $c_{0}=c_{3}=0$. Тогда для решения уравнения (8) получим

$$
y(x, \lambda)=-\frac{1}{2 i \lambda} \int_{-\infty}^{+\infty} G(x, t, \lambda) f(t) d t+\left\{\begin{array}{ll}
c_{1} f_{-}(x, \lambda), & x \in(-\infty, 0), \\
c_{2} f_{+}(x, \lambda), & x \in(0,+\infty),
\end{array} \quad \lambda \in S_{\nu}\right.
$$

при

$$
G(x, t, \lambda)= \begin{cases}f_{+}(x, \lambda) f_{-}(t, \lambda), & t \leqslant x \\ f_{-}(x, \lambda) f_{+}(t, \lambda), & t \geqslant x\end{cases}
$$

Здесь числа $c_{j}, j=\overline{1,2}$, определяются из условий (9), а именно

$$
\begin{gathered}
c_{1} f_{-}(0, \lambda)-c_{2} f_{+}(0, \lambda)=0 \\
c_{1}\left[f_{-}^{\prime}(0, \lambda)-\beta f_{-}(0, \lambda)\right]-c_{2} f_{+}^{\prime}(0, \lambda)=-\frac{\beta}{2 i \lambda} \int_{-\infty}^{+\infty} G(0, t, \lambda) f(t) d t .
\end{gathered}
$$

Отсюда

$$
\begin{gathered}
c_{1,2}=-\frac{\beta f_{ \pm}(0, \lambda)}{2 i \lambda D(\lambda)} \int_{-\infty}^{+\infty} G(0, t, \lambda) f(t) d t \\
D(\lambda)=-\left|\begin{array}{cc}
f_{-}(0, \lambda) & f_{+}(0, \lambda) \\
f_{-}^{\prime}(0, \lambda)-\beta f_{-}(0, \lambda) & f_{+}^{\prime}(0, \lambda)
\end{array}\right| .
\end{gathered}
$$

В результате решение задачи (8), (9) принимает вид

$$
\begin{aligned}
y(x, \lambda)= & -\frac{1}{2 i \lambda} \int_{-\infty}^{+\infty} G(x, t, \lambda) f(t) d t+ \\
& +\frac{\beta}{2 i \lambda D(\lambda)} \begin{cases}f_{+}(0, \lambda) f_{-}(x, \lambda) \int_{-\infty}^{+\infty} G(0, t, \lambda) f(t) d t, & x \in(-\infty, 0), \\
f_{-}(0, \lambda) f_{+}(x, \lambda) \int_{-\infty}^{+\infty} G(0, t, \lambda) f(t) d t, & x \in(0, \infty) .\end{cases}
\end{aligned}
$$

Tеорема 2. Спектр операторного пучка L состоит из непрерывного спектра, заполняющего ось $\{-\infty<\lambda<+\infty\}$, на которой могут бить спектральные особенности, совпадающие с числами вида $\pm n / 2, n \in N$, и не более одного собственного числа $\lambda_{1}$, которое определяется как корень уравнения $D(\lambda)=0$.

ДокАЗАТЕЛьСтво. Известно [6], что при $\beta=0$ спектр операторного пучка $L$ (обозначим его через $\left.L_{0}\right)$ является чисто непрерывным и заполняет ось $\{-\infty<\lambda<+\infty\}$, на которой могут быть спектральные особенности, совпадаюшие с числами вида $\pm n / 2$, $n \in N$. Согласно работе [7] спектр оператора $L$ может отличаться от спектра операторного пучка $L_{0}$ разве что на конечное число собственных значений. Кроме того, число этих собственных значений не превосходит ранг оператора, $R_{\lambda}-R_{\lambda(\beta=0)}$, т.е. числа 1 . Таким образом, теорема доказана. 


\section{3. ОБРАТНАЯ ЗАДАЧА}

Из соотношения (7) видно, что числа $S_{n}^{ \pm}$играют роль "нормировочных" чисел для функций, отвечаюших спектральным особенностям $( \pm n / 2), n=1,2, \ldots$.

Ставится задача определения функций $q(x), p(x)$ и $\beta$ по числам $\left\{S_{n}^{ \pm}\right\}$. Вначале найдем явные связи между последовательностями $\left\{S_{n}^{ \pm}\right\}$и $\left\{V_{n \alpha}^{ \pm}\right\},\left\{V_{n}^{ \pm}\right\}$. Для этого воспользуемся тождествами (7) в развернутом виде:

$$
\sum_{\alpha=m}^{\infty} V_{n \alpha}^{ \pm} e^{i \alpha x} e^{-i(n / 2) x}=S_{n}^{ \pm} e^{i(n / 2) x}\left(1+\sum_{n=1}^{\infty} V_{n}^{\mp} e^{i n x}+\sum_{n=1}^{\infty} \sum_{\alpha=n}^{\infty} \frac{V_{n \alpha}^{\mp}}{n+n} e^{i \alpha x}\right) .
$$

При этом находим, что

$$
V_{m m}^{ \pm}=S_{m}^{ \pm}, \quad V_{m, \alpha+m}^{ \pm}=S_{m}^{ \pm}\left(V_{\alpha}^{\mp}+\sum_{n=1}^{\alpha} \frac{V_{n \alpha}^{\mp}}{n+m}\right),
$$

а $\beta$ определим с помощью равенства

$$
\beta=-\frac{y^{\prime}(+0)-y^{\prime}(-0)}{y(0)} .
$$

Эти соотношения являются основными уравнениями для определения $\left\{q_{\alpha}\right\},\left\{p_{\alpha}\right\}$ и $\beta$ по числам $\left\{S_{n}^{ \pm}\right\}$.

ТЕОРЕМа 3. Для того чтобы числа $\left\{S_{n}^{ \pm}\right\}$были “нормировочнылми" числами операторного пучка типа L с потенциалами вида (2), достаточно, чтобы выполнялись условия

$$
\sum_{m=1}^{\infty} m\left|S_{m}^{*}\right|=\delta<\infty, \quad \sum_{m=1}^{\infty} \frac{\left|S_{m}^{*}\right|}{1+m}=p<1,
$$

əде $\left|S_{m}^{*}\right|=\max \left\{\left|S_{m}^{+}\right|,\left|S_{m}^{-}\right|\right\}$.

\section{Список литературы}

[1] С. Альбеверио, Ф. Гастези, Р. Хеәг-Крон, Х. Хольден. Решаемые модели в квантовой механике. М.: Мир, 1991.

[2] P. C. Sabatier. J. Math. Phys. 1968. V. 9. № 8. P. 1241-1258.

[3] Р. И. Кадиев (мл.). Изв. вузов. Математика. 1998. № 7. С. 26-31.

[4] T. Aktosun. Inverse Problems. 2004. № 20. P. 859-876.

[5] Р. Ф. Эфендиев. Докл. НАН Азерб. 2001. № 4-6. С. 15-20.

[6] Р. Ф. Эфендиев. Матем. физика, анализ, геометрия. 2004. Т. 11. № 1. С. 114-121.

[7] П. Н. Князев. Изв. вузов. Математика. 1959. № 2. С. 94-100.

Поступила в редакцию 20.XII.2004 г., после доработки 6.IV.2005 г. 